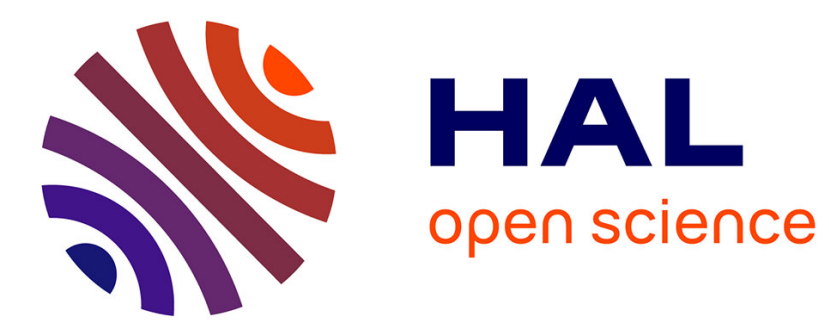

\title{
Correlated field evaporation as seen by atom probe tomography
}

\author{
F. de Geuser, B. Gault, A. Bostel, F. Vurpillot
}

\section{To cite this version:}

F. de Geuser, B. Gault, A. Bostel, F. Vurpillot. Correlated field evaporation as seen by atom probe tomography. Surface Science: A Journal Devoted to the Physics and Chemistry of Interfaces, 2007, 601 (2), pp.536 - 543. 10.1016/j.susc.2006.10.019 . hal-00408087

\section{HAL Id: hal-00408087 https://hal.science/hal-00408087}

Submitted on 29 Jul 2009

HAL is a multi-disciplinary open access archive for the deposit and dissemination of scientific research documents, whether they are published or not. The documents may come from teaching and research institutions in France or abroad, or from public or private research centers.
L'archive ouverte pluridisciplinaire HAL, est destinée au dépôt et à la diffusion de documents scientifiques de niveau recherche, publiés ou non, émanant des établissements d'enseignement et de recherche français ou étrangers, des laboratoires publics ou privés. 


\title{
Correlated field evaporation as seen by atom probe tomography
}

\author{
Frédéric De Geuser ${ }^{\mathrm{a}, \mathrm{b}, *}$ Baptiste Gault ${ }^{\mathrm{a}}$ Alain Bostel ${ }^{\mathrm{a}}$ \\ François Vurpillot ${ }^{\mathrm{a}}$ \\ ${ }^{\mathrm{a}}$ GPM - UMR 6634 CNRS - Université et INSA de Rouen, 76801 Saint-Etienne du \\ Rouvray Cedex, FRANCE \\ ${ }^{\mathrm{b}}$ ALCAN Centre de Recherches de Voreppe - BP 27, 38241 Voreppe Cedex - FRANCE
}

\begin{abstract}
We have studied the correlation between field evaporated atoms during an atom probe tomography experiment. The evaporated atoms have been shown to be highly correlated both in time and in space. This correlation can be explained by the dynamic distribution of the electric field at the surface of the sample. The evaporation sequence follows the distribution of zones where the electric field intensity is higher. The distance of correlation between successively evaporated atoms corresponds to the mean size of these zones and the time of correlation corresponds to the mean time necessary to evaporate the entire zone.
\end{abstract}

Key words: Atom probe tomography, field evaporation, simulation

\section{Introduction}

The last twenty years have seen the emergence of a new class of analytical techniques in materials science. The Atom Probe Tomography enables the three dimensional visualization of chemical composition variations with a near atomic spatial resolution in small volumes of materials [1]. This technique is of great interest in the study of the early stages of phase transformations, surface and interface segregations, and more generally in nanotechnology applications [2,3]. The basic principle of the technique is the pulsed field evaporation of specimen atoms and their detections by a position sensitive detector with a high performance timing measurement

* Corresponding author. Present address: ARC Centre of Excellence for Design in Light Metals, Monash University, Clayton 3800, Victoria, Australia.

Tel.: +61 39905 3827; Fax.: +61 39905 4940;

E-mail: frederic.degeuser@eng.monash.edu.au 
accuracy. Chemical natures of detected ions from the probed volume are deduced by time-of-flight mass spectrometry.

The first generation of instrument, the position sensitive atom probe (PoSAP) was developed at the end of the 80's by Cerezo and co-workers [4]. This instrument gave the first qualitative 3D images of various metallurgical cases. In this instrument, however, difficulties arises when more than one ion is evaporated from the probed area on a single evaporation pulse. Indeed, if two ions arrive simultaneously at the detector, their positions cannot be determined. This problem limits the accuracy of analyses performed on the resultant data. In order to avoid significant loss of data from the analysis, one solution is to decrease the mean rate of evaporation (down to 0.001 atom/pulse) to automatically reduce the statistical occurrence of multi-hits events. This is simply done by decreasing the electric field at the tip surface. However this decrease can result in undesirable effects such as field corrosion or preferential retention. Furthermore, it was shown recently that even at a low detection rate, the occurrence of multi-hit events is still important [5]. This prevents this type of instrument to obtain quantitative measurement for materials science applications.

To overcome these drawbacks, other instruments based on the use of detection system capable of multi-hit detection were developed. These instruments have proved to generally give reliable composition measurements [6] when the flight length of the instrument is not too short $(>20 \mathrm{~cm})$. The problem observed experimentally is that multi-hit events are shown to be highly correlated in time and in space. The ability to detect multi-hit events is thus related to the spatial and time resolving powers of the position sensitive detector.

The correlation between hits on the detector is related to the process of field evaporation itself. It indicates that atoms close to each other on the tip surface are often evaporated on the same evaporation pulse, whatever the electric field at the tip surface. The correlated evaporation was studied in field ion microscopy $[7,3]$. By studying the sequence of evaporation of surface atoms with this instrument, simultaneous evaporation is often observed. This phenomenon is particularly drastic at the centre of low index poles. Such types of burst evaporation are thought to be mainly due to the increase in electric field in proportion as the pole terrace diameter decreases. As field evaporation has a really critical dependance on the electric field, it is evident that the sequence of evaporation of the atoms can not be completely random, as it has been observed in field ion microscopy [8] or even in atom probe tomography $[9,10]$.

In this paper, the co-evaporation has been studied experimentally using atom probe tomography and the results have been compared with a simple analytical model and numerical simulation [11,12]. Both models are based on basic principles of field evaporation. 


\section{Atom probe tomography (APT)}

The atom probe tomography is based on the field evaporation of specimen surface atoms. The high electric field required is obtained by applying a high voltage to the sample prepared in the form of a sharply pointed needle with a end radius in the range 10-100 $\mathrm{nm}$. The tip is biased at a high positive DC voltage in the range 1$20 \mathrm{kV}$. Surface atoms are field evaporated by means of electric pulses superimposed on the DC voltage $\mathrm{V}_{0}$ with a pulse fraction $\left(\mathrm{V}_{\mathrm{p}} / \mathrm{V}_{0}\right)$ close to $20 \%$. The electric field $\mathrm{F}$ at the tip surface is directly related to the total applied voltage $\mathrm{V}$ and is inversely proportional to the curvature radius $\mathrm{R}$ of the tip apex $(F=V / \beta R$ with $\beta$ a constant related to the shape of the specimen and the surrounding electrostatic environment). The field necessary for evaporation is thus approached only during the time of the evaporation pulses $(\approx 1 \mathrm{~ns})$ defining a precise start time for the time-of-flight mass spectrometer.

Field evaporated atoms (ions) are rapidly accelerated and projected onto the positionsensitive detector (PSD). The magnification is due to the diverging electric field that is present in the vicinity of the end apex of the tip specimen. The image transfer function is generally assumed to be a simple point projection. As the specimen is evaporated atom by atom, layer by layer, a 3D reconstruction of the analyzed volume can be made. The depth co-ordinate is deduced from the amount of materials evaporated. The spatial resolution of the instrument was proven to achieve less than a few angstroms.

\section{Basics of the field evaporation of metals}

\section{$3.1 \quad$ Field evaporation process}

Field evaporation $[13,14]$, refers to the process of removing an ion from a fieldemitter surface with a high electric field. The required field intensity is in the range $10-60 \mathrm{~V} / \mathrm{nm}$ for metal tip emitters. Theory of field evaporation is now fairly well developed. The basic concepts of field evaporation are best explained with reference to one-dimensional potential energy diagrams [15]. In the absence of electric field, neutral states are generally the stable states at the surface of materials. In the presence of a high electric field, ionic states will become more stable as the distance of the atom from the surface is increased. It is therefore possible for an atom to become an ion by crossing the barrier formed by the atomic and ionic potentials. The potential energy barrier is reduced by the application of the electric field.

Atoms are generally field evaporated in a thermal process. The potential energy barrier is defined by $Q_{n}$ depending on the heat of sublimation of a neutral surface 
atom, the ionisation energy of this atom and the work function of an electron at the surface. The rate constant $K_{e}$ for field evaporation by thermal activation over the barrier is simply given by an Arrhenius expression:

$$
K_{e}=\mathrm{v}_{n} \cdot \exp \left(\frac{-Q_{n}}{k_{B} T}\right)
$$

Where $v_{n}$ is the surface atom vibration frequency, $k_{B}$ the Boltzmann constant and $T$ the applied temperature. At low temperature $(<100 \mathrm{~K})$, the rate of evaporation is mainly controlled by the intensity of the electric field at the tip surface. If the electric field is raised to a value close to a threshold value named field evaporation $F_{e}$, atoms are field evaporated in an exponential manner. In a first approach, $Q_{n}$ is thus considered generally as a linear function of the electric field $F$. At a constant temperature and close to the evaporation field $F_{e}$, the rate of evaporation is

$$
K_{e} \propto \exp \left(a \cdot\left(\frac{F-F_{e}}{F_{e}}\right)\right)
$$

With $a$ a constant. For instance, at $80 \mathrm{~K}$ the evaporation rate of metals may change by a factor of 10 for a $2 \%$ change in field strength [3]. The constant a is thus about 115. The mean time required for evaporation of a surface atom may thus be written

$$
\tau \propto \exp \left(-a \cdot\left(\frac{F-F_{e}}{F_{e}}\right)\right)
$$

The evaporation rate may be carefully controlled so only a few atoms may be removed from the surface. The position sensitive detector intercepts a restricted area of analysis, so that only a fraction of the evaporated atoms are detected. Considering that the number of surface atoms is approximately constant during the analysis (i.e. the radius of curvature changes slowly), the detection rate $K_{D}$ is also defined by the same type of relationship.

$$
K_{D} \propto \exp \left(a \cdot\left(\frac{F-F_{e}}{F_{e}}\right)\right)
$$

Considering all the atoms of the surface, the mean time required to detect an ion is

$$
\tau_{D} \propto \exp \left(-a \cdot\left(\frac{F-F_{e}}{F_{e}}\right)\right)
$$

As the evaporation field is only reached during the electric pulses, $\tau_{D}$ only runs during the application of the pulses. 


\subsection{Waiting time of evaporation and multiple events occurrence}

In a simple approach, as the evaporation is thermally activated, one can consider the evaporation to be a stochastic process at low evaporation rate. The waiting time $\tau$ between two successive events is therefore related to a Poisson process and the probability distribution function $P_{w}(\tau)$ to get a waiting time $\tau$ can be statistically defined by an exponential law

$$
P_{w}(\tau)=\frac{1}{\tau_{D}} \exp \left(-\frac{\tau}{\tau_{D}}\right)
$$

For low probability events (which is true at a mean detection rate $K_{D}<0.1$ atom per pulse), the probability of detecting $n$ atoms $P_{\text {multi }}(n)$ on the time of a single pulse therefore follows a Poisson law

$$
P_{\text {multi }}(n)=\frac{K_{D}^{n}}{n !} \exp \left(-K_{D}\right)
$$

Following this equation, the probability $P_{m u l t i}(n>1)$ to detect a multi-hit is therefore

$$
\begin{aligned}
P_{\text {multi }}(n>1) & =1-P_{\text {multi }}(0)-P_{\text {multi }}(1) \\
& =1-\exp \left(-K_{D}\right)-K_{D} \exp \left(-K_{D}\right)
\end{aligned}
$$

With low evaporation rates $(<0.1$ atom/pulse), the probability to detect more than one ion should be lower than $1 \%$. Most of the evaporation pulses give rise to 0 or 1 detected ion.

We define the time of evaporation $\tau_{e}$ as the pulse duration during which evaporation is possible. $\tau_{e}$ is related to the detection rate $K_{D}$ by

$$
\tau_{e}=\tau_{D} \cdot K_{D}
$$

As $\tau_{e}$ is a constant related to the evaporation pulse duration ( $\left.<1 \mathrm{~ns}\right)$, the number of pulses between two successive events is directly linked to the waiting time $\tau$ between two events. As a result, the probability density functional $P_{\text {pulses }}\left(n_{p}\right)$ to get a number of pulses $n_{p}$ between two successive impacts can be written the same way as eq. (6)

$$
P_{\text {pulses }}\left(n_{p}\right)=\frac{1}{\overline{n_{p}}} \exp \left(-\frac{n_{p}}{\overline{n_{p}}}\right)
$$

with $\overline{n_{p}}$ the mean number of pulses between two impacts. Note that $\overline{n_{p}}$ is also the 
inverse of the mean detection rate $K_{D}$ so that

$$
P_{\text {pulses }}\left(n_{p}\right)=K_{D} \exp \left(-n_{p} \cdot K_{D}\right)
$$

This type of probability treatments have been successfully applied in the past to $1 \mathrm{D}$ atom probe data in order to compute the effect of the Poissonian process of field evaporation on the measured composition of alloys [16] or to detect fine scale clustering by studying the succession of detected atoms [17].

\subsection{Distance between impacts}

If the evaporation is a thermally activated process, one can consider that atoms are picked randomly onto the surface of analysis. This implies that, in the event of the detection of more than one impact on a single pulse, the repartition of the impacts on the detection surface $S_{A}$ is uniform and that the position of one of the detected impacts is independent from the position of the other impacts.

Let us consider a pulse giving rise to the detection of $n$ distinct impacts. The number $N_{i-j}^{n}$ of pairs $i-j(i, j=1,2, \ldots n$ and $i \neq j)$ is equal to $n \cdot(n-1)$. We can now multiply this number by the probability of detecting $n$ impacts (eq. (7)). The sum of this expression on each value of $n(n>1)$ gives the mean number of impacts pairs per pulse :

$$
\begin{aligned}
N_{i-j} & =\sum_{n=2}^{\infty} N_{i-j}^{n} P_{\text {multi }}(n) \\
& =\sum_{n=2}^{\infty} n \cdot(n-1) \frac{K_{D}^{n}}{n !} \exp \left(-K_{D}\right) \\
& =K_{D}^{2} \overbrace{\sum_{u=0}^{\infty} \frac{K_{D}^{u}}{u !} \exp \left(-K_{D}\right)}^{=1} \\
N_{i-j} & =K_{D}^{2}
\end{aligned}
$$

As the repartition of the impacts is uniform, the surface density of impacts pairs $\sigma$ is constant on the surface of analysis and $\sigma=K_{D}^{2} / S_{A}$. In the case of an infinite surface of analysis, the probability $P_{d}\left(R_{D}\right)$ of measuring a distance $\left[R_{D}, R_{D}+\Delta R_{D}\right]$ between two impacts is simply equal to the product of the surface density $\sigma$ by the surface of a ring of radius $R_{D}$ and of thickness $\Delta R_{D}$, thus

$$
P_{d}\left(R_{D}\right)=\sigma 2 \pi R_{D} \Delta R_{D}=\frac{K_{D}^{2} 2 \pi R_{D} \Delta R_{D}}{S_{A}}
$$


The histogram of distances between impacts follows a linear law. Although eq. (13) is theoretically only valid for an infinite surface of analysis, it is possible to use it for a finite surface provided that periodic boundary conditions are used when computing the distances histogram. In these conditions, eq. (13) is valid for $R_{D}$ inferior to a maximum distance $R_{D}^{\max }$ that corresponds to the surface of analysis $S_{A}$ $\left(S_{A}=\pi\left(R_{D}^{\max }\right)^{2}\right.$ or $\left.R_{D}^{\max }=\sqrt{S_{A} / \pi}\right)$.

\section{Numerical modelisation of the evaporation process}

A model was recently developed to reproduce either the electric field distribution and field evaporation process at the apex of a realistic specimen or to simulate the aberrations of ion trajectories in the atom probe. In this model, the tip was modelled as a 3D compact stack of polygons (Wigner-Seitz cells). These cells are initially confined inside a hemisphere. The atom centre is suppose to coincide with the centre of the cell. The tip is (001) orientated, and has a radius of curvature of the order of $10 \mathrm{~nm}$.

Through a finite elements method, the Laplace equation $(\Delta V=0)$ is numerically solved, and the equipotentials are thus determined. Since the evaporation is highly sensitive to the electric field, the atom submitted to the highest electic field was chosen to be evaporated. Its trajectory is calculated towards a virtual detector placed near the tip. Once this atom removed from the surface, both potential and electric field distributions were recalculated, so that the dynamic electric field variations were taken into account in the model.

This very simple procedure was found to be very consistent with experimental evidence of the order existing in the field evaporation. The kink site atoms are firstly evaporated, as submitted to the highest electric field. The tip is layer by layer evaporated. Using this model it was shown that a stationary state can be achieved [12]. Evaporation was shown to be homogenous at the tip surface, and the distribution of the electric field during the evaporation was found to converge to a narrow distribution centered on the evaporation field. The equilibrium surface of the sample was shown to be facetted following some crystallographic directions. The shape of the surface, together with the electric field precise distribution also helped with the interpretation of FIM contrasts [11]. The numerical model has also been previously used to understand the trajectory aberrations in atom probe tomography (APT) [18].

\section{Experimental behaviour of the evaporation process}

Experimental datasets were obtained on aluminium based alloys with the Rouen Tomographic Atom Probe (TAP) instrument. This instrument has a $45 \mathrm{~cm}$ flight 

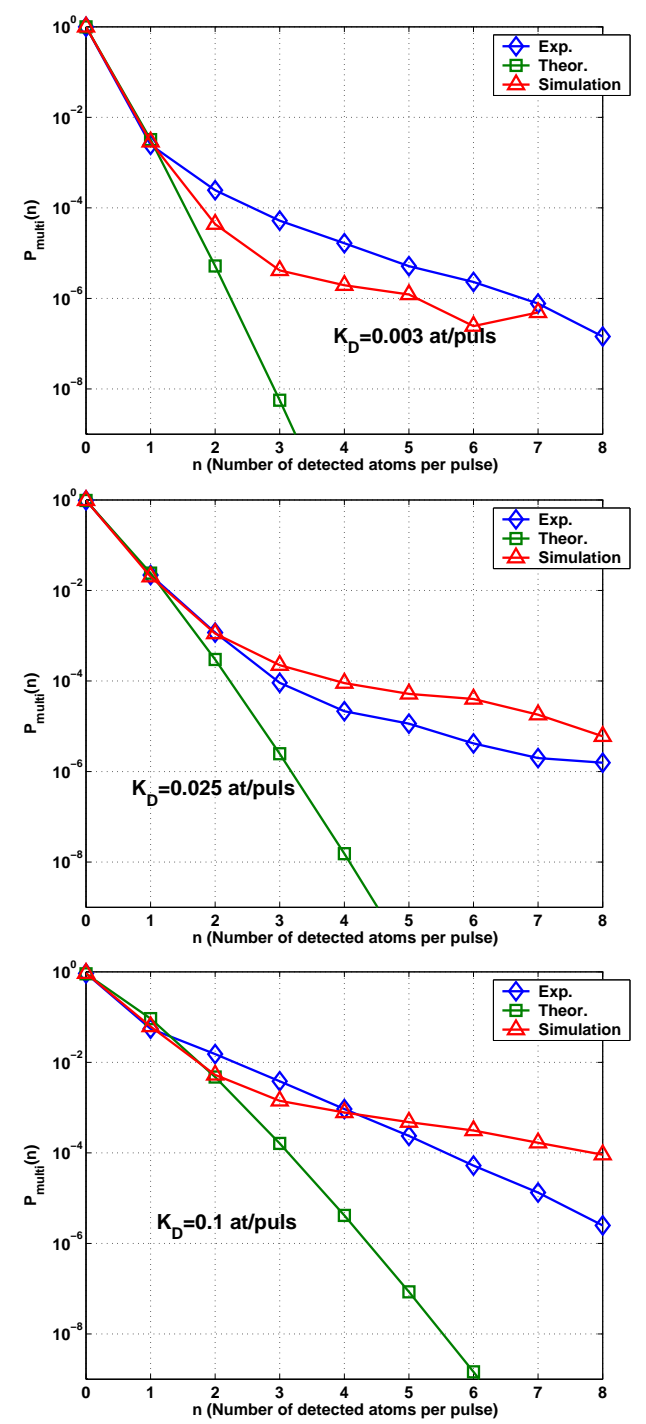

Fig. 1. Experimental histograms of the number of detected atoms per pulse compared to the theoretical Poisson law. a) 0.003 atom/pulse, b) 0.025 at/pulse and c) 0.1 at/pulse. The results of our simulations are also shown(red crosses).

length and the detector is a $10 \times 10 \mathrm{~cm}^{2}$ position sensitive detector with multi-hits capability. The sample temperature was kept constant $(80 \mathrm{~K})$. The three different probability laws $P_{\text {multi }}, P_{\text {pulses }}$ and $P_{d}$ (eq. (7), (11) and (13)) can be extracted from experimental datasets. An experimental raw dataset consists in a list of events that are filed according to the detection order. The number of pulses between successive events, the number of impacts, the mass-to-charge ratios and the $2 \mathrm{D}$ positions are stored in the file. Using these informations, probability laws were experimentally measured on various datasets.

Histograms of the number of atoms per pulse are drawn in figure 1 for three different detection rates $(0.003$ atom/pulse, 0.025 atom/pulse and 0.1 atom/ pulse). Voltage ranges of $5-7 \mathrm{kV}$ were used during experiments. The experimental histograms 

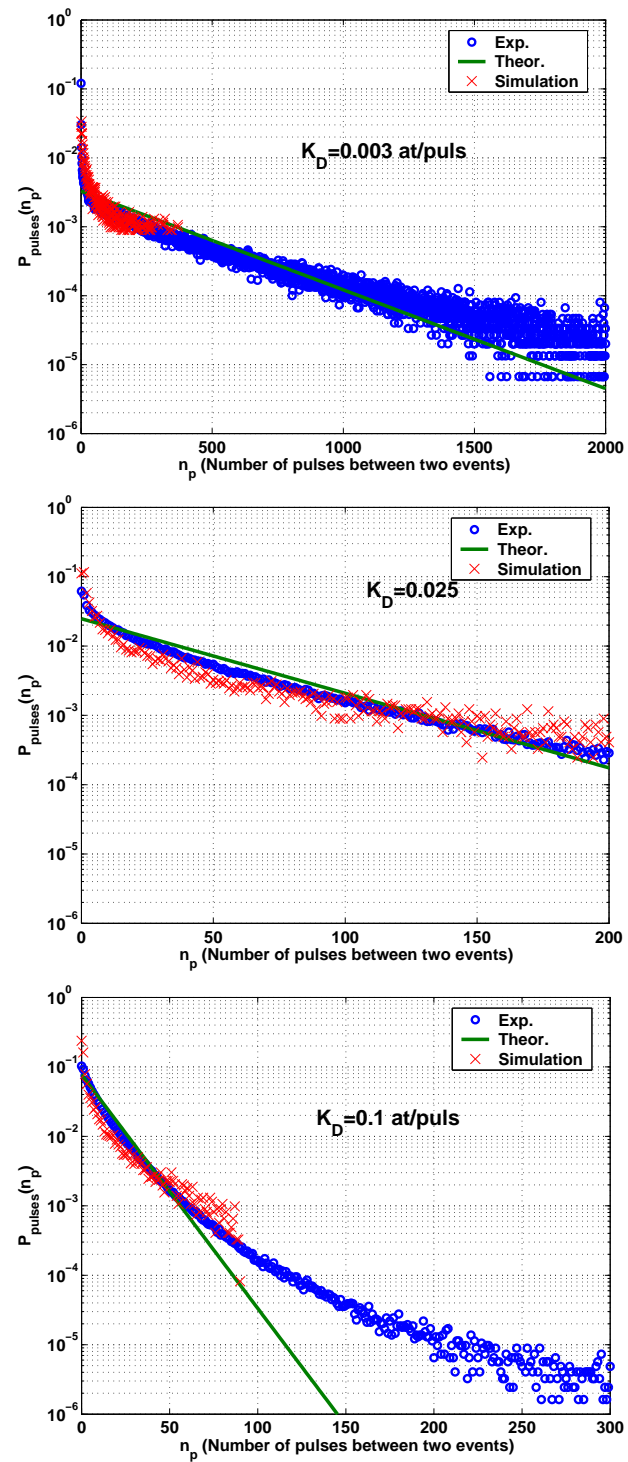

Fig. 2. Experimental histograms of the number of pulses between two detection events. a) $0.003 \mathrm{at} / \mathrm{pulse}$, b) $0.025 \mathrm{at} / \mathrm{pulse}$ and c) $0.1 \mathrm{at} / \mathrm{pulse}$. Note the difference of scale in the figure. The results of our simulations are also shown(red crosses).

were compared to Poisson law with identical detection rates. Whereas the number of single hit events is found in good agreement with Poisson behaviour whatever the detection rate, strong differences are observed for multi-hits events. The experimental distribution differs strongly from the Poisson law with the increasing number of pulses. It is worth noting that the difference is stronger for low evaporation rate. The number of events with multiple impacts (up to 20) is kept high even with very low evaporation rate. This behaviour was observed experimentally whatever the temperature of the specimen, the nature of the analysed material or the voltage of the tip. Note that this phenomenon was found worse in alloys where different elemental species with distinct evaporation field are present. 


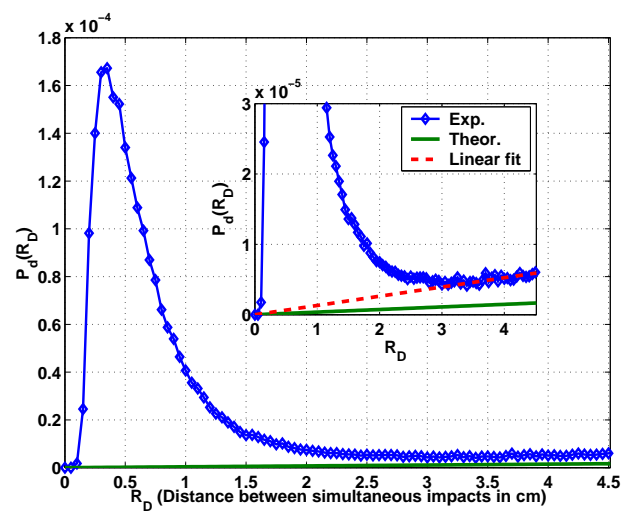

Fig. 3. Experimental histograms of the distance between simultaneous impacts on the detector. The inset shows the random (linear) contribution.

This behaviour is also observed experimentally when considering the waiting time of evaporation. The theoretical probability density functional $P_{\text {pulses }}\left(n_{p}\right)$ to get a number of pulses $n_{p}$ between two successive detection events is compared to experimental law in figure 2. As predicted theoretically, the probability to get $n_{p}$ pulses between two events decays strongly with $n_{p}$. The experimental curves follows an exponential decrease with high number of pulses. However, at low pulses number, the experimental curves diverge from this behaviour. Impacts are found to be detected preferentially separated by a small number of pulses.

These two results show that the evaporation at the tip surface does not follow a random statistical behaviour. The evaporation of successive atoms is found to be strongly correlated in time. This correlation prevents the decrease of multi-hit events with very low evaporation rate.

The probability functional to get a distance $d$ between two successive impacts can also be extracted from the position information stored in the file. The distance between simultaneous impacts is derived from their relative positions. In order to avoid artefacts due to the limited area of the detector, periodic boundary conditions were applied to the dataset. The figure 3 presents a typical experimental probability functional $P_{d}$ compared to the theoretical variation law. If a random (linear) contribution exists (see inset, Fig. 3), the divergence with the random law for short distances is evident.

In order to study specifically the correlated part of the histograms, it is possible to isolate it from the random contribution by subtracting a linear fit of the end of the distribution. Furthermore, it is possible to transform the distances between impacts on the detector (with no particular physical meaning) in distances between atoms at the surface of the tip. To do this, one needs to compute the magnification which is a function of the radius of curvature of the sample apex and is routinely used for atom probe tomography experiments. This transformation has been done on the same aluminium based alloy. Two analyses were performed on the same sample, but on different crystallographic locations ([210] and [100], see Fig. 4). In both 


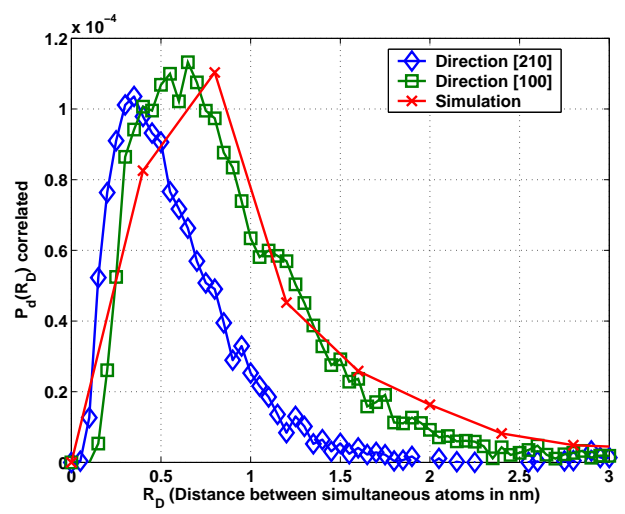

Fig. 4. Correlated contribution of the histograms of distances between simultaneously detected atoms for two crystallographic analysis locations. The results of our simulations are also shown(red crosses). Please note that the simulated lattice structure (simple cubic) is different than that of aluminium (fcc).

cases, a hump in the distribution is observed for short distances between impacts. The shape of this hump, however, is found different in the two crystallographic locations.

This result demonstrates that there is a correlation in space between evaporated atoms. The difference in the hump positions strongly suggests that the correlation is linked to the crystallographic structure of the analysed surface. The aluminium [210] crystallographic pole is known to be brightly imaging in field-ion microscopy (FIM) and gives rise to the thinnest hump in the distribution of distances, whereas the [100] pole, darkly imaging in FIM, shows a broader distances distribution. The relation between the contrast in FIM and the thickness of the correlated distance distribution is a serious evidence of the existence of a relation between the electric field distribution at the surface of the tip and the correlation between the evaporated atoms.

\section{Modelling the co-evaporation process}

As the last paragraph states, it is most likely that the correlation between the atoms are related to the dynamic distribution of the electric field on the surface of the sample. Considering equation (2), the evaporation process is a stochastic process governed by the temperature but also by the exact electric field that is applied to atoms to be evaporated. This electric field is dependent on the voltage applied to the tip, but also on the local curvature which is not homogeneous at the whole surface. The mean shape of the tip is known to be facetted, low index pole regions are generally flatter that high index pole regions, giving rise to local variations of electric field. More fundamentally, the shape of the tip is not smooth at the atomic level. The tip surface shape is modulated by the presence of atoms. 
In order to understand the co-evaporation process, one needs knowledge of the electric field modulation at the tip surface at the atomic scale. A simple description of the tip shape as a smooth hemispherical shape or other model considering the tip at a mesoscopic scale is no longer sufficient. Moreover, the electric field intensity at the tip surface changes as the tip is evaporated atom after atom. This means that a correct model must take into account the gradual change of the tip morphology at the atomic scale.

The model described in section 4 can be used successfully to understand the coevaporation process as it implies a computation of the local electric field applied to each surface atom. As already said, it is most likely that the correlation in time and in space is related to the distribution and the dynamic fluctuations of the electric field at the surface. Once the tip has reached a steady state, the mean shape of the tip does not evolve anymore. However, this is only true at a mesoscopic scale.

If, at a given time, we observe the evaporation of a sequence of 100 atoms successively evaporated, we see that they originate from defined zones of the sample surface (Fig. 5). These 100 atoms are among the atoms submitted to the highest electric field. We can see that these atoms are either at the edge of atomic terraces or situated on scattered limited zones.

This is due by the fact that the atom positions in these zones are somehow equivalent in regard to the electric field, but also by the increase in electric field caused by the evaporation of one atom on its neighbours. If on a mesoscopic scale the evaporation of a local amount of material leads to an increase of the local radius of curvature and therefore to the decrease of the surface electric field, on an atomic scale, the evaporation of a single atom can generate a local increase of the electric field above surrounding neighbours and consequently to the local evaporation of several atoms. This explains the correlation in time and in space between the successively evaporated atoms.

Only a slight modification to the model is necessary to take the co-evaporation process into account in the evaporation sequence. According to eq. (5), the waiting time between two events is related to the electric field. In our model, we compute the electric field above each surface atom before and after each evaporation. It is, thus, possible to compute the waiting time between two successive atoms by using the electric field applied to the second atom (before its evaporation) in eq. (5). The waiting time between two evaporation is related to the number of pulses $n_{p}$ between two atoms by $\tau_{D}=\tau_{e} \cdot n_{p}$, thus

$$
n_{p}=\frac{\alpha}{\tau_{e}} \exp \left(-a \frac{F-F_{e}}{F_{e}}\right)
$$

Where $\alpha$ is a proportionality factor. When $n_{p}$ is inferior to 1 , it means that the atom has been evaporated on the same pulse than the first one. When $n_{p}$ is superior to 


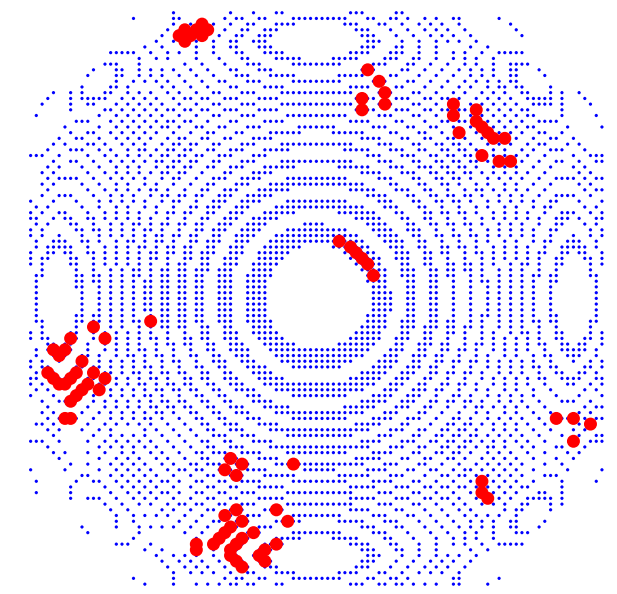

Fig. 5. Surface of our simulated sample (blue dots) at a given time. The red circles are the next 100 atoms in the evaporation sequence.

1 , the actual number of pulses between the two atoms is the entire part of $n_{p}$. The proportionality factor $\alpha$ is adjusted for getting the expected mean detection rate $K_{D}$. By recording the value of $n_{p}$ for each evaporated atom in the sequence, it is possible to compare the correlation of the successively evaporated atoms in our model and in the experiments.

An interesting property of eq. 14 is the fact that the number of pulses between two events and consequently the number of atoms detected per pulse do not depend directly on the time of evaporation $\tau_{e}$, but on the ratio $\alpha / \tau_{e}$. This means that, to reduce the co-evaporation process, it is useless to reduce the pulse duration, as only $K_{D}$ the detection rate per pulse has an influence on it.

We have compared our simulations with both experimental data and the theoretical model based on the absence of correlation between the evaporated atoms. Concerning the histograms of the number of detected atoms per pulse (Fig. 1), the simulations histograms agree reasonably well with the experimental one. The differences can be explained differently depending on the considered detection rate $K_{D}$. For the two highest detection rate $\left(K_{D}=0.1\right.$ at/pulse and $K_{D}=0.025$ at/pulse), the lower experimental values for large numbers of detected atoms per pulse can be explained by the finite efficiency of the detection system. For the lowest detection rate $\left(K_{D}=0.003 \mathrm{at} / \mathrm{pulse}\right)$, the difference between experimental data and simulated data goes the other way round. This is a serious hint for the existence of other correlation factors not included in our model and which are of bigger influence at low detection rate.

To illustrate the fact that our simulations also reproduce the correlation in space between the evaporated atoms, the figure 4 also presents the correlated part of the histogram of distances between simultaneous atoms. It is worth noting, however, that the space correlation is directly related with the crystallographic location of the analysis and the lattice structure used for the simulations (simple cubic) is different 
than that of the aluminium (fcc), although their lattice parameters are the same.

The comparison between the simulated and experimental histograms of the number of pulses between two events (Fig. 2) is even more interesting,as there are no experimental bias due to the finite efficiency of the detector. Concerning these histograms, the simulations data are in very good agreement with the experiments.

\section{Discussion}

The atoms that are field evaporated during an atom probe tomography (or FIM) analysis have been shown to be highly correlated in time and in space. Our simulations show that this phenomenon can be explained by a non uniform distribution of the electric field at the surface of the tip. The atoms that are simultaneously evaporated are situated in regions of the surface where the electric field is similar. When the surface has reached its equilibrium shape, there are several atom positions on a tip surface that are completely equivalent regarding the electric field. Moreover, considering the order of evaporation, the evaporation of one atom can lead to the rapid evaporation of surrounding neighbours. This correlated evaporation is due to the discrete nature of the geometrical surface. The evaporation of one atomic volume modifies the local roughness of the surface. Surrounding atoms are submitted to a high electric field and are evaporated swiftly. This explains the fact that simultaneous impacts are often situated very close to each other, and that the distribution of the distances between impacts presents a maximum that is very close to the first neighbours distance on a particular crystallographic terrace.

The strong divergence with the Poisson law for the histogram of the number of atoms detected per pulse (Fig. 1) can be very well understood by this concept of local dynamic variation of regarding the electric field. On a particular pulse, if an atom is likely to evaporate because of its electric field higher than the mean electric field of the surface, its evaporation can increase the probability to evaporate local neighbours. This will increase strongly the probability detection of more than one atom per pulse as compared to a Poisson law.

Concerning the histograms of number of pulses between two events (Fig. 2), although the dynamic distribution of the electric field at the surface of the tip explains the type of figure that are observed, it is worth noting that other phenomenons can be invoked. In particular, we implicitly supposed that the electric potential applied to the sample was stable in time. Unfortunately, as the instrument is working at high voltage and the pulses are of very short duration, the complexity of the circuit can lead to instabilities in the intensity of the pulses. In particular, the pulses immediately following a detection can be significantly more intense. This could lead to the detection of more atoms distant from small number of pulses than expected. The resulting curves would then be very similar to Fig. 2. In this case, however, 
there will be no reason for finding any correlation in space between the detected atoms. The existence of this space correlation strongly indicates that, although the instabilities of the electric potential intensity might exist, their effect is clearly not predominant.

The correlation in time and space between the field evaporated atoms can, thus, be explained both by the fact that, at a given time, there are definites zones in which all the atoms are likely to evaporate, and by the fact that the evaporation of one atom increase the electric field applied on its neighbours.

It is interesting to note that the co-evaporation process does not only involve atoms detected on the same evaporation pulse. The co-evaporation process have been explained in the past by phenomena happening during the pulse. Even if this can happen, our results show that it does not seem to be the predominant cause for co-evaporation. Indeed, the field evaporated atoms are still correlated even if separated by several pulses (Fig. 2 shows that the correlation can extend to hundreds of pulses). The type of correlation is the same between atoms evaporated on the very same pulse or between atoms separated by one or a few pulses. The histogram of distances between atoms separated by several pulses (not shown here) are of the same type than Fig. 3 and Fig. 4.

The strong correlation in time and space between the field evaporated atoms is of particular interest in the development of detection systems for atom probe tomography instruments. Although all the existing instruments have a multi-hit detection system, their multi-hit detection capacities are not always the same. While evaluating the actual detection loss induced by the correlated evaporation, it is very important not to use random distribution laws, neither in time nor in space. Indeed, whereas a Poisson law will predict that the detection of more than two impacts is a very rare events, our experimental distributions (i.e. distribution that are already underestimated because of the non-perfect detection system) show that this event can involve more than $10 \%$ of the total number of detected atoms even at a detection rate as low as 0.003 at. per pulse. Furthermore, this possible decrease of the theoretical detection efficiency is most likely to affect particularly the different chemical species, leading to specific loss, and thus alter the chemical composition measurements.

\section{References}

[1] D. Blavette, A. Bostel, J. Sarrau, B. Deconihout, and A. Menand, Nature 363, 432 (1993).

[2] D. Blavette, E. Cadel, A. Fraczkiewicz, and A. Menand, Science, 286, 5448 (1999).

[3] M. Miller, A. Cerezo, M. Hetherington, and G. Smith, Atom Probe Field Ion Microscopy, Oxford Science Publications, Clarendon press edition, (1996). 
[4] A. Cerezo, T.J. Godfrey, and G.D.W. Smith Rev. Sci. Instrum. 59, 862 (1988).

[5] L. Renaud PhD Thesis, Université de Rouen (2001).

[6] D. Blavette, B. Deconihout, A. Bostel, J.M. Sarrau, M. Bouet, and A. Menand, Rev. Sci. Instrum. 64, 2911 (1993).

[7] K.M. Bowkett and D.A. Smith North-Holland Publishing Company, (1970).

[8] A.J.W. Moore, and J.A. Spink Surf. Sci. 12, 479 (1968).

[9] J.M. Hyde, A. Cerezo, R.P. Setna, P.J. Warren and G.D.W. Smith Appl. Surf. Sci. 76, 382 (1994).

[10] P. Bas, A. Bostel, G. Grancher, B. Deconihout, and D. Blavette Appl. Surf. Sci. 94, 442 (1996).

[11] F. Vurpillot, A. Bostel, A. Menand, and D. Blavette Eur. Phys. J. Appl. Phys. 6, 217 (1999).

[12] F. Vurpillot, A. Bostel, and D. Blavette J. of Mic. 196, 332 (1999).

[13] E. Muller Naturwissenschaften 29, 533 (1941).

[14] E. Muller, Z. Physik, 131,136 (1951).

[15] T.T. Tsong Atom probe field ion microscopy, Cambridge University press, (1990).

[16] T.T. Tsong, Yee S. Ng and S.V. Krishnaswamy Appl. Phys. Lett. 32, 778 (1978).

[17] T.T. Tsong, S.B. McLane Jr., M. Ahmad and C.S. Wu J. Appl. Phys. 53, 4180 (1982).

[18] F. Vurpillot, A. Bostel and D. Blavette, Appl. Phys. Lett. 76, 3127 (2000). 